\title{
A Comparison of the DES and Dömösi Cryptosystems
}

\section{Zoltan Pal Mecsei}

Research Group on Mathematical Linguistics

Universitat Rovira i Vigili

Tarragona, Spain

E-mail: zoltan.mecsei@estudiants.urv.cat

Summary. In this paper we compare the well known DES cryptosystem with the recently introduced Dömösi system, which is based on finite automata. We do a time complexity analysis on both algrithms. We show that without making use of an auxiliary matrix the Dömösi cryptosystem is slower than DES. However, the use of auxiliary matrices makes the former perform better than its well known counterpart for some block lengths.

\section{The Data Encryption Standard (DES)}

First let us take a look at the DES cryptosystem. In particular, if we consider the following to be elementary steps (es): reading input, comparing two values, jumping, the system will have the following requirements:

1. First we perform the initial permutatincludegraphicion as seen in Figures 1 and 2. This phase consists of 64 elementary steps.

2. a) Make a copy of the current half of the 64 bits (1 es)

b) Extend the current 32 bits to 48 bits (48 es) (Figure 3) 


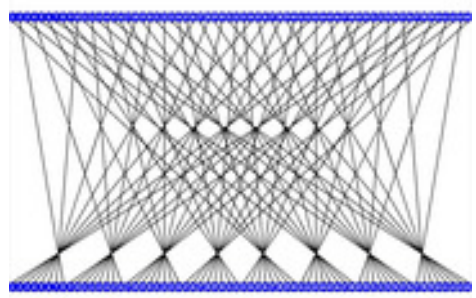

Fig. 1. Initial permutation

\begin{tabular}{|c|c|c|c|c|c|c|c|}
\hline 58 & 50 & 42 & 34 & 26 & 18 & 10 & 2 \\
\hline 60 & 52 & 44 & 36 & 28 & 20 & 12 & 4 \\
\hline 62 & 54 & 46 & 38 & 30 & 22 & 14 & 6 \\
\hline 64 & 56 & 48 & 40 & 32 & 24 & 16 & 8 \\
\hline 57 & 49 & 41 & 33 & 25 & 17 & 9 & 1 \\
\hline 59 & 51 & 43 & 35 & 27 & 19 & 11 & 3 \\
\hline 61 & 53 & 45 & 37 & 29 & 21 & 13 & 5 \\
\hline 63 & 55 & 47 & 39 & 31 & 23 & 15 & 7 \\
\hline
\end{tabular}

Fig. 2. IP

\begin{tabular}{|c|c|c|c|c|c|}
\hline 32 & 1 & 2 & 3 & 4 & 5 \\
\hline 4 & 5 & 6 & 7 & 8 & 9 \\
\hline 8 & 9 & 10 & 11 & 12 & 13 \\
\hline 12 & 13 & 14 & 15 & 16 & 17 \\
\hline 16 & 17 & 18 & 19 & 20 & 21 \\
\hline 20 & 21 & 22 & 23 & 24 & 25 \\
\hline 24 & 25 & 26 & 27 & 28 & 29 \\
\hline 28 & 29 & 30 & 31 & 32 & 1 \\
\hline
\end{tabular}

Fig. 3. Expansion function (E)

c) Read the first key (1 es)

d) XOR the 48 bits resulting from $2 \mathrm{~b}$ with the key read (1 es)

e) do 8 s-box $6 \mapsto 4$ bit mappings $(3 \times 8=24$ es) (Figure 4 )

f) apply the 32 bit permutation (32 es) (Figure 5)

g) XOR what we have so far and the remaining 32 bits (Figure 6)

3. Swap the two sides (1 es)

4. Perform the inverse permutation (64 es) as seen in Figures 7 and 8

We can now summarize the calculations on each of the steps. More exactly we will get the following: 


\begin{tabular}{|c|c|c|c|c|c|c|c|c|c|c|c|c|c|c|c|}
\hline 14 & 4 & 13 & 1 & 2 & 15 & 11 & 8 & 3 & 10 & 6 & 12 & 5 & 9 & 0 & 7 \\
\hline 0 & 15 & 7 & 4 & 14 & 2 & 13 & 1 & 10 & 6 & 12 & 11 & 9 & 5 & 3 & 8 \\
\hline 4 & 1 & 14 & 8 & 13 & 6 & 2 & 11 & 15 & 12 & 9 & 7 & 3 & 10 & 5 & 0 \\
\hline 15 & 12 & 8 & 2 & 4 & 9 & 1 & 7 & 5 & 11 & 3 & 14 & 10 & 0 & 6 & 13 \\
\hline
\end{tabular}

Fig. 4. Substitution box (S-box) $S_{1}$

\begin{tabular}{|c|c|c|c|}
\hline 16 & 7 & 20 & 21 \\
\hline 29 & 12 & 28 & 17 \\
\hline 1 & 15 & 23 & 26 \\
\hline 5 & 18 & 31 & 10 \\
\hline 2 & 8 & 24 & 14 \\
\hline 32 & 27 & 3 & 9 \\
\hline 19 & 13 & 30 & 6 \\
\hline 22 & 11 & 4 & 25 \\
\hline
\end{tabular}

Fig. 5. Permutation $(\mathrm{P})$

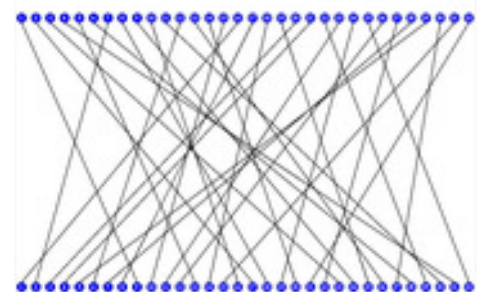

Fig. 6. XOR with the remaining 32 bits

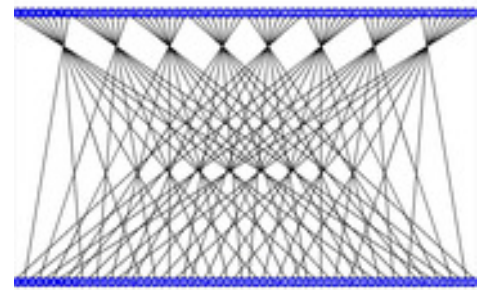

Fig. 7. Final permutation $\left(I P^{-1}\right)$ 


\begin{tabular}{|c|c|c|c|c|c|c|c|}
\hline 40 & 8 & 48 & 16 & 56 & 24 & 64 & 32 \\
\hline 39 & 7 & 47 & 15 & 55 & 23 & 63 & 31 \\
\hline 38 & 6 & 46 & 14 & 54 & 22 & 62 & 30 \\
\hline 37 & 5 & 45 & 13 & 53 & 21 & 61 & 29 \\
\hline 36 & 4 & 44 & 12 & 52 & 20 & 60 & 28 \\
\hline 35 & 3 & 43 & 11 & 51 & 19 & 59 & 27 \\
\hline 34 & 2 & 42 & 10 & 50 & 18 & 58 & 26 \\
\hline 33 & 1 & 41 & 9 & 49 & 17 & 57 & 25 \\
\hline
\end{tabular}

Fig. 8. Inverse of IP

1 ststep :

$64 e s$

2ndstep :

$(1+48+1+1+24+32+1) \times 16=1728 e s$

3rdstep :

4thstep :

$64 e s$

The number of steps needed to perform the 64-bit DES are given by the table in Figure 9. Summing up this table gives us a total of 1857 steps. For a detailed and more formal time complexity analysis of the algorithm please refer to $[3,4,5]$.

\begin{tabular}{|c|c|c|c|c|}
\hline Step & Operation & Time & $\begin{array}{c}\text { Equivalent } \\
\text { total }\end{array}$ & Notes \\
\hline 1. & IP 64 bit transposition & 1 & 64 & \\
\hline 2.a & 32 bit Copy & 16 & 16 & *16 steps \\
\hline $2 . \mathrm{b}$ & 48 bit transposition & 16 & $48 \times 16$ & $*$ \\
\hline 2.c & READ the key & 16 & 16 & * \\
\hline $2 . \mathrm{d}$ & 48 bit XOR & 16 & 16 & * \\
\hline $2 . \mathrm{e}$ & $\begin{array}{c}6 \mapsto 4 \text { bit two } \\
\text { dimensional mapping }\end{array}$ & $8 \times 16$ & $3 \times 128$ & * \\
\hline $2 . f$ & 32 bit transposition & 16 & $32 \times 16$ & * \\
\hline $2 . \mathrm{g}$ & 32 bit XOR & 16 & 16 & * \\
\hline 3. & 32 bit swapping & 1 & 1 & \\
\hline 4. & $I P^{-1}$ & 1 & 64 & \\
\hline
\end{tabular}

Fig. 9. Number of steps required by DES 


\section{The Dömösi System}

Let us now move on to the Dömösi system and take a look at the number of elementary steps needed to process 64 bits with or without using an auxiliary matrix.

\subsection{Without auxiliary matrix}

As we move on to the Dömösi system first we will take a look at the number of elementary steps needed to process 64 bits without using an auxiliary matrix. When considering a Dömösi system without an auxiliary matrix, from a final state we will have the following phases to follow:

1. Read a character $8 \times[1]$, where the number between [] is the number of elementary steps; read a character from previously generated random number row $8 \times[1]$; in the transition matrix we find the state transition corresponding to the random number read $8 \times[$ Psurit $]$, where Psurit is the number of elementary steps of the logical and physical correspondence for the transition matrix

2. This phase is a longer one that depends on the length $k$ of the given code word. The expected step count is $8 \times k \times[2 \times$ Psurit +4$]$

3. The expected cost of the 3 rd step is $8 \times 2 \times$ Psurit +4 .

4. Now that the parity is correct, we have to look for the input sign which will take the automaton into a final state. Based on the reference we can expect that this goes down in $8 \times[128 \times$ Psurit +132$]$ steps, that is with 4 "if"s and with final state compression and comparison reachable in 128 steps.

The phases are explicitly described in the Figure 10:

These four cases consist altogether of $k$ steps of wandering, parity change and state identifying. Together with the cost of the first steps this gives a total of elementary steps described by:

$$
8 \times[\text { Psurit } \times(2 k+131)+144+k \times 4]
$$

If we suppose $c$ to be the extra cost for every $k$ steps, in other words the upper approximation for the cost of the extra steps performed by the algorithm, then the formula is equivalent to $8 \times[$ Psurit $\times(2 k+131)+144+k \times(4+c)]$. When implementing in Windows, this extra cost is large for any algorithm, depending on Windows' event handler and scheduler. However, for the purpose of theoretical time complexity analysis we can disregard this cost, as it is insignificant compared to the other factors.

Comparison: In the case of the Dömösi system, the processing of 8-byte generating code words of length $k$ takes $8 \times($ Psurit $\times(2 k+131)+144+k \times(4+c))$ elementary steps. Furthermore, we can take Psurit to be equal to 3 (as it is considered in the Figure 10) and $c$ to be 0 . This way the time cost of processing a 8-byte DES block becomes: 


\begin{tabular}{|c|c|c|c|c|}
\hline Step & Operation & Times & Equivalent total & Notes \\
\hline $1 \mathrm{a}$ & Read a character & 8 & 8 & \\
\hline $1 \mathrm{~b}$ & $\begin{array}{l}\text { Read the next } \\
\text { random value }\end{array}$ & 8 & 8 & \\
\hline $1 \mathrm{c}$ & Mapping & 8 & 8*Psurit & \\
\hline $2 \mathrm{a}$ & $\begin{array}{l}\text { Read the next } \\
\text { random value }\end{array}$ & $8 * k^{*} 2$ & $8 * k^{*} 2$ & $\begin{array}{l}\text { *probably } \\
\text { in two steps }\end{array}$ \\
\hline $2 \mathrm{~b}$ & Mapping & $8 * k^{*} 2$ & $8 * \mathrm{k}^{*} 2 *$ Psurit & $\begin{array}{l}\text { *probably } \\
\text { in two steps }\end{array}$ \\
\hline $2 \mathrm{c}$ & $\begin{array}{l}\text { Comparison(random } \\
\text { in non-final state) }\end{array}$ & $8 * k^{*} 2$ & $8 * k^{*} 2$ & $\begin{array}{l}\text { *probably } \\
\text { in two steps }\end{array}$ \\
\hline $3 a$ & $\begin{array}{l}\text { Read the next } \\
\text { random value }\end{array}$ & $8 * 2$ & $8 * 2$ & $\begin{array}{l}\text { *probably } \\
\text { in two steps }\end{array}$ \\
\hline $3 \mathrm{~b}$ & Mapping & $8 * 2$ & $8 * 2 *$ Psurit & $\begin{array}{l}{ }^{*} \text { probably } \\
\text { in two steps }\end{array}$ \\
\hline $3 \mathrm{c}$ & $\begin{array}{c}\text { Comparison (random in } \\
\text { non-final state } \\
\text { with correct parity) }\end{array}$ & $8^{*} 2$ & $8 * 2$ & $\begin{array}{l}\text { *probably } \\
\text { in two steps }\end{array}$ \\
\hline $4 a$ & Checking & 8 & $8 * 4$ & *check the cases \\
\hline $4 \mathrm{~b}$ & $\begin{array}{l}\text { Mapping to the } \\
\text { right final state }\end{array}$ & $8 * 128$ & $8 * 128 *$ Psurit & $\begin{array}{c}\text { *probably } \\
\text { in } 128 \text { steps }\end{array}$ \\
\hline $4 \mathrm{c}$ & $\begin{array}{l}\text { Comparison (map } \\
\text { value, final state) }\end{array}$ & $8 * 128$ & $8 * 128$ & $\begin{array}{l}{ }^{*} \text { probably } \\
\text { in } 128 \text { steps }\end{array}$ \\
\hline
\end{tabular}

Fig. 10. No auxiliary matrix

$$
8 \times(3 \times(2 k+131)+144+k \times 4))=10 k+537
$$

By looking at the ratio $80 k+4296 / 1857$ we see that without the auxiliary matrix the algorithm is slower than the DES.

\subsection{With auxiliary matrix}

In this section we compare the DES cryptosystem with the Dömösi system that uses auxiliary matrices.

With the introduction of the auxiliary matrix the cost becomes minimal in Case 4 , as it can be seen in the table. In the case of the DES this cost is 6 . Thus, the formula is transformed into Psurit $\times(3+2 k)+4 k+12+6$. Considering once more Psurit $=3$ we get $80 k+168=1857$. Since the equality holds for $k \approx 21$, it follows that for block lengths 21 the Dömösi cryptosystem with auxiliary matrix performs at same level as the DES cryptosystem. Thus, for blocks of shorter length the Dömösi 


\begin{tabular}{|c|c|c|c|c|}
\hline Step & Operation & Times & Equivalent total & Notes \\
\hline $1 \mathrm{a}$ & Read a character & 8 & 8 & \\
\hline $1 \mathrm{~b}$ & $\begin{array}{l}\text { Read the next } \\
\text { random value }\end{array}$ & 8 & 8 & \\
\hline $1 c$ & Mapping & 8 & $8 *$ Psurit & \\
\hline $2 \mathrm{a}$ & $\begin{array}{l}\text { Read the next } \\
\text { random value }\end{array}$ & $8 * k^{*} 2$ & $8 * \mathrm{k}^{*} 2$ & $\begin{array}{l}* \text { probably } \\
\text { in two steps }\end{array}$ \\
\hline $2 \mathrm{~b}$ & Mapping & $8 * k^{*} 2$ & $8 * \mathrm{k}^{*} 2 *$ Psurit & $\begin{array}{l}{ }^{*} \text { probably } \\
\text { in two steps }\end{array}$ \\
\hline $2 \mathrm{c}$ & $\begin{array}{c}\text { Comparison(random } \\
\text { in non-final state) }\end{array}$ & $8 * \mathrm{k}^{*} 2$ & $8 * k^{*} 2$ & $\begin{array}{l}* \text { probably } \\
\text { in two steps }\end{array}$ \\
\hline $3 a$ & $\begin{array}{l}\text { Read the next } \\
\text { random value }\end{array}$ & $8 * 2$ & $8 * 2$ & $\begin{array}{l}* \text { probably } \\
\text { in two steps }\end{array}$ \\
\hline $3 \mathrm{~b}$ & Mapping & $8 * 2$ & $8 * 2 *$ Psurit & $\begin{array}{l}* \text { probably } \\
\text { in two steps }\end{array}$ \\
\hline $3 \mathrm{c}$ & $\begin{array}{l}\text { Comparison (random } \\
\text { in non-final state } \\
\text { with correct parity) }\end{array}$ & $8^{*} 2$ & $8 * 2$ & $\begin{array}{l}* \text { probably } \\
\text { in two steps }\end{array}$ \\
\hline $4 a$ & Checking & 8 & $8 * 4$ & *check the cases \\
\hline $4 \mathrm{~b}$ & $\begin{array}{l}\text { Mapping to the } \\
\text { right final state }\end{array}$ & $8 * 6$ & $8 * 6 *$ Psurit & $\begin{array}{c}\text { Exactly } \\
\text { in } 6 \text { steps } \\
\end{array}$ \\
\hline
\end{tabular}

Fig. 11. Using auxiliary matrices

cryptosystem is faster than DES while, naturally, when taking longer blocks it is slower. It is still a question, how secure the system remains when assuming these block lengths.

\section{References}

1. Dömösi, P. (2007). Symmetric key cryptographic method and apparatus for information encryption and decryption.

2. Dömösi, P. (2007). A practical stream cipher based on finite automata without outputs, manuscript.

3. Elkeelany, O., M.M. Matalgah, K.P Sheikh, M. Thaker, G. Chaudhry, D. Medhi and J. Qaddour (2002). Performance analysis of IPSec protocol: encryption and authentication. Communications, 2002. ICC 2002. IEEE International Conference, volume 2, pp. 1164-1168.

4. http://en.wikipedia.org/wiki/DES_supplementary_material

5. http://en.wikipedia.org/wiki/Data_Encryption_Standard\#Overall_structure 